\title{
Relative Fatou theorem for harmonic functions of rotation invariant stable processes in smooth domains
}

\author{
by \\ Krzysztof Bogdan and BartŁomiej Dyda (Wrocław)
}

\begin{abstract}
For $C^{1,1}$ domains we give exact asymptotics near the domain's boundary for the Green function and Martin kernel of the rotation invariant $\alpha$-stable Lévy process. We also obtain a relative Fatou theorem for harmonic functions of the stable process.
\end{abstract}

1. Introduction. The composition of a Markov process with a nonnegative function harmonic for the process (for definitions see Preliminaries) is a nonnegative supermartingale. By Doob's theory of martingales the supermartingale has limits at the lifetime of the process. When the lifetime coincides with the exit time of a domain, an important problem arises how to interpret this probabilistic convergence in analytical or topological terms involving only the function and the domain but not the process.

Such Fatou theorems $[\mathrm{F}]$ were first studied for classical harmonic functions, the Brownian motion $W_{t}$ being the underlying Markov process; see, e.g., [S], [Ca] for purely analytical exposition, [D2] for the probabilistic counterpart, and $[\mathrm{BM}],[\mathrm{Ba}]$ for both approaches.

The continuous paths of $W_{t}$ leave the domain by hitting the boundary and, typically, do not creep along the boundary before the exit time [BaB]. This explains why for harmonic functions the notion of nontangential convergence at the domain's boundary $([\mathrm{S}],[\mathrm{Wu} 1])$ is an appropriate counterpart of the martingale convergence for the Brownian motion and other diffusion processes. A natural problem in a more general framework is to formulate relevant analytical counterparts of the martingale convergence theorems for discontinuous (jump) processes. Such processes typically leave a sufficiently regular domain without approaching its boundary by jumping to the interior of its complement [Mi], [Sz]; see also [Wu2] in this connection. In that case Doob's theory does not admit a natural and interesting topological interpre-

2000 Mathematics Subject Classification: Primary 31B25, 60J45. function.

Key words and phrases: relative Fatou theorem, symmetric stable process, $\alpha$-harmonic

Partially supported by KBN grant 2P03A 04122 and RTN grant RTN2-2001-00315. 
tation even though some quantitative analytical results are still available. In this connection we refer the reader to the paper $[\mathrm{BaC}]$, devoted to the study of the boundary behavior of the symmetric stable Lévy processes $X_{t}$ in the unit ball of $\mathbb{R}^{d}$.

The subject of the present paper is the same stable process $X_{t}$ and its boundary behavior on $C^{1,1}$ domains in $\mathbb{R}^{d}$ (see below for the definitions). However, to obtain a nontrivial result on the boundary convergence of harmonic functions of $X_{t}$, we apply Doob's $h$-transform to the process or, what is the same, we normalize the harmonic functions considered by dividing them by a suitable harmonic function $h$. We prove the existence of nontangential limits of such quotients at the domain's boundary.

Such relative Fatou theorems ([D1], [Wu1]) depend on the approximate factorization of the Martin kernel of the domain. This factorization is related ([B3], [J]) to the boundary Harnack principle for nonnegative harmonic functions [Da], [Wu1], [A], [JK]; see also [B1] for the case of harmonic functions of the stable process $X_{t}$.

Doob's $h$-process converges to the set of poles of the harmonic function $h$. Thus the discontinuous stable process $X_{t}$ may be conditioned to leave the domain by hitting its boundary (see, e.g., [CZ], [BB2]). For $C^{1,1}$ (more generally, Lipschitz) domains the appropriate class of $h$ functions is spanned by the Martin kernel of the domain with poles at the boundary introduced in [B2], [CS3]. Such functions are called singular $\alpha$-harmonic (see below for a definition), and they are harmonic for the stable process killed on leaving the domain rather than for the process stopped at the exit time [CS3]; see [MS] for the general representation theorem for $\alpha$-harmonic functions and [B2] for the special case of Lipschitz domains.

There is a growing literature on fine properties of $\alpha$-harmonic functions and other potential properties of the stable process $X_{t}$ (see, e.g., [BB3], $[\mathrm{BKN}],[\mathrm{M}],[\mathrm{BBC}])$. In this paper we depend on the estimates of the Martin and Green functions of $C^{1,1}$ domains obtained in [K], [CS1], [CS2]. The estimates as well as other recent developments owe much to the explicit calculation of the Green function and the Martin kernel of the ball by M. Riesz [R1], [R2] (see also [BGR]).

In the present paper we do not use probabilistic techniques in an essential way, keeping them only for interpretation as above. Our focus is on expressing explicitly the asymptotic behavior of the relevant harmonic functions in $C^{1,1}$ domains in terms of the distance to the boundary. In Section 2 we introduce the notation used in this paper. In Section 3 we give the exact asymptotics of the Green function of $C^{1,1}$ domains. We note that the main result of this section, Theorem 3.2, is stated in [CS1] but no detailed proof is given there. Our methods are different from those used in [CS1]. They are also simpler and in Section 4 we can pursue our asymptotic investigation fur- 
ther to obtain Theorem 4.3, which is our second main technical result. The relative Fatou theorem for $\alpha$-harmonic functions is given in Theorem 4.2. The theorem answers a question of Jang-Mei Wu about $C^{1,1}$ domains and confirms a hypothesis of Renming Song. Still open remains the case of Lipschitz domains, which is perhaps the most natural setting for this problem.

2. Preliminaries. We denote by $|\cdot|$ the Euclidean length of a vector. For $A \subset \mathbb{R}^{d}$ we put $A^{\mathrm{c}}=\left\{x \in \mathbb{R}^{d}: x \notin A\right\}$. For $x \in \mathbb{R}^{d}, r>0$ and a set $A \subset \mathbb{R}^{d}$ we define $B(x, r)=\left\{y \in \mathbb{R}^{d}:|x-y|<r\right\}$ and $\delta_{A}(x)=\inf \left\{|x-y|: y \in A^{\mathrm{c}}\right\}$.

We write $D$ for a nonempty open bounded set in $\mathbb{R}^{d}$. We assume that $D$ is a $C^{1,1}$ domain, i.e., there exist $r_{0}>0$ and $\lambda>0$ such that for each $Q \in \partial D$ there are a $C^{1,1}$ function $\varphi: \mathbb{R}^{d-1} \rightarrow \mathbb{R}$ and an orthonormal coordinate system $y=\left(y_{1}, \ldots, y_{d}\right)$ satisfying

$$
D \cap B\left(Q, r_{0}\right)=\left\{y: y_{d}>\varphi\left(y_{1}, \ldots, y_{d-1}\right)\right\} \cap B\left(Q, r_{0}\right),
$$

and, furthermore, $\nabla \varphi$ is Lipschitz with Lipschitz constant not greater than $\lambda$.

For the rest of the paper, unless stated otherwise, $\alpha$ is a number in $(0,2)$ and $d=1,2, \ldots$ We denote by $\left(X_{t}, \mathbf{P}_{x}\right)$ the standard [BG] rotation invariant ("symmetric") $\alpha$-stable, $\mathbb{R}^{d}$-valued Lévy process (i.e. homogeneous, with independent increments) with stability index $\alpha$ and with characteristic function

$$
\mathbf{E}_{x} e^{i \xi\left(X_{t}-x\right)}=e^{-t|\xi|^{\alpha}}, \quad x \in \mathbb{R}^{d}, \xi \in \mathbb{R}^{d}, t \geq 0 .
$$

For a Borel set $U \subset \mathbb{R}^{d}$, we put $\tau_{U}=\inf \left\{t \geq 0: X_{t} \notin U\right\}$, the first exit time of $U$. Given $x \in \mathbb{R}^{d}$, the $\mathbf{P}_{x}$ distribution of $X_{\tau_{U}}$ is a subprobability measure on $U^{\text {c }}$ (probability measure if $U$ is bounded) called the $\alpha$-harmonic measure.

When $r>0,|x|<r$ and $B=B(0, r) \subset \mathbb{R}^{d}$, the corresponding $\alpha$-harmonic measure has the density function $P_{r}(x, \cdot)$ (the Poisson kernel) given by

$$
P_{r}(x, y)=C_{\alpha}^{d}\left[\frac{r^{2}-|x|^{2}}{|y|^{2}-r^{2}}\right]^{\alpha / 2}|y-x|^{-d} \quad \text { if }|y|>r,
$$

with $C_{\alpha}^{d}=\Gamma(d / 2) \pi^{-d / 2-1} \sin (\pi \alpha / 2)$, and 0 otherwise. The formula for the Poisson kernel for the exterior of the ball, $\left\{y \in \mathbb{R}^{d}:|y-x|>r\right\}$, is similar. Namely, for $|x|>r$ we have

$$
\widetilde{P}_{r}(x, y)=C_{\alpha}^{d}\left[\frac{|x|^{2}-r^{2}}{r^{2}-|y|^{2}}\right]^{\alpha / 2}|y-x|^{-d} \quad \text { if }|y|<r,
$$

and $\widetilde{P}_{r}(x, y)=0$ if $|y| \geq r$. Both (1) and (2) can be found in [BGR].

We say that a function $f$ defined on $\mathbb{R}^{d}$ is $\alpha$-harmonic in an open set $D \subset \mathbb{R}^{d}$ if it has the "mean value property" 


$$
f(x)=\mathbf{E}_{x} f\left(X_{\tau_{U}}\right), \quad x \in U,
$$

for every bounded open set $U$ with closure contained in $D$. It is called regular $\alpha$-harmonic in $D$ if (3) holds for $U=D$ and singular $\alpha$-harmonic if $f=0$ on $D^{\mathrm{c}}$.

By the strong Markov property a regular $\alpha$-harmonic function is $\alpha$ harmonic. The converse is not generally true as shown in [B2], [CS3]. An alternative description of $\alpha$-harmonic functions as those annihilated by the fractional Laplacian is given in [BB3]. It follows from (1) and (3) that an $\alpha$-harmonic function $f$ in $D$ satisfies

$$
f(x)=\int_{|y-\theta|>r} P_{r}(x-\theta, y-\theta) f(y) d y, \quad x \in B(\theta, r),
$$

for every ball $B(\theta, r)$ with closure contained in $D$. Note that if $f$ is nonzero in $D$, then it is positive in $D$, regardless of the connectedness of $D$. This is a consequence of the Harnack inequality (see, e.g., [BB1]).

We write $G_{D}(\cdot, \cdot)$ for the Green function of $D$, i.e.

$$
\mathbf{E}_{x} \int_{0}^{\tau_{D}} f\left(X_{t}\right) d t=\int G_{D}(x, y) f(y) d y
$$

for all nonnegative Borel measurable functions $f$ (see $[\mathrm{K}]$, $[\mathrm{L}]$ ). Recall from [BGR] that

$G_{B(0, r)}(x, y)=\mathcal{B}_{d, \alpha}|x-y|^{\alpha-d} \int_{0}^{w(x / r, y / r)} \frac{s^{\alpha / 2-1}}{(s+1)^{d / 2}} d s, \quad x, y \in B(0, r), x \neq y$, and

$$
G_{B(0, r)^{\mathrm{c}}}(x, y)=\mathcal{B}_{d, \alpha}|x-y|^{\alpha-d} \int_{0}^{w(x / r, y / r)} \frac{s^{\alpha / 2-1}}{(s+1)^{d / 2}} d s, \quad x, y \in B(0, r)^{\mathrm{c}}, x \neq y,
$$

where $w(x, y)=\left(1-|x|^{2}\right)\left(1-|y|^{2}\right) /|x-y|^{2}, \mathcal{B}_{d, \alpha}=2^{-\alpha} \pi^{-d / 2} \Gamma(\alpha / 2)^{-2} \Gamma(d / 2)$.

We denote by $M^{x_{0}}(\cdot, \cdot)$ the Martin kernel of $D$ based at $x_{0} \in D$ :

$$
M^{x_{0}}(x, Q)=\lim _{D \ni y \rightarrow Q} \frac{G_{D}(x, y)}{G_{D}\left(x_{0}, y\right)}, \quad x \in \mathbb{R}^{d}, Q \in \partial D .
$$

The existence of the above limit follows from the boundary Harnack principle (see [B1]). There are constants $c$ and $C$, depending only on $D, \alpha$ and $x_{0}$, such that

$$
c \frac{\delta_{D}(x)^{\alpha / 2}}{|x-Q|^{d}} \leq M^{x_{0}}(x, Q) \leq C \frac{\delta_{D}(x)^{\alpha / 2}}{|x-Q|^{d}}
$$

for all $x \in D$ and $Q \in \partial D$ (see [CS3]).

Every nonnegative function singular $\alpha$-harmonic on $D$ has a unique representation

$$
f(x)=\int_{\partial D} M^{x_{0}}(x, Q) \mu(d Q), \quad x \in \mathbb{R}^{d}
$$


where $\mu$ is a finite nonnegative measure on $\partial D$. Conversely, for every signed measure $\mu$ on $\partial D$ the formula (6) defines a (singular) $\alpha$-harmonic function on $D$ (see [B2], [CS3]).

\section{The Green function. We define}

$$
f_{r}(x, y)=\left.\frac{2}{\alpha} \mathcal{B}_{d, \alpha} r^{-\alpha}|x-y|^{-d}|| x\right|^{2}-\left.r^{2}\right|^{\alpha / 2}(|y|+r)^{\alpha / 2}
$$

for all $x, y \in \mathbb{R}^{d}, x \neq y$.

Lemma 3.1. For $x, y \in B(0, r), x \neq y$, we have

$$
\frac{f_{r}(x, y)}{(w(x / r, y / r)+1)^{d / 2}} \leq \frac{G_{B(0, r)}(x, y)}{\delta_{B(0, r)}(y)^{\alpha / 2}} \leq f_{r}(x, y),
$$

and for $x, y \in \operatorname{int} B(0, r)^{\mathrm{c}}, x \neq y$, we have

$$
\frac{f_{r}(x, y)}{(w(x / r, y / r)+1)^{d / 2}} \leq \frac{G_{B(0, r)^{\mathrm{c}}}(x, y)}{\delta_{B(0, r)^{\mathrm{c}}}(y)^{\alpha / 2}} \leq f_{r}(x, y) .
$$

Proof. For $x, y \in B(0, r), x \neq y$, we have

$$
\begin{aligned}
\frac{G_{B(0, r)}(x, y)}{\delta_{B(0, r)}(y)^{\alpha / 2}} & =\mathcal{B}_{d, \alpha}|x-y|^{\alpha-d}|| y|-r|^{-\alpha / 2} \int_{0}^{w(x / r, y / r)} \frac{s^{\alpha / 2-1}}{(s+1)^{d / 2}} d s \\
& \leq \mathcal{B}_{d, \alpha}|x-y|^{\alpha-d}|| y|-r|^{-\alpha / 2} \int_{0}^{w(x / r, y / r)} s^{\alpha / 2-1} d s=f_{r}(x, y) .
\end{aligned}
$$

We obtain the lower bound similarly. The same proof works for the second part of the lemma.

Note that

$$
\lim _{D \ni y \rightarrow Q} \frac{G_{B(0, r)}(x, y)}{\delta_{B(0, r)}(y)^{\alpha / 2}}=f_{r}(x, Q)
$$

and similarly for $G_{B(0, r)^{c} \text {. }}$

Now we will introduce some notation. Our assumptions on $D$ imply existence of an $R>0$ with the following property: for every $z \in \partial D$ there are two balls $B(z) \subset D$ and $E(z) \subset D^{\mathrm{c}}$ with radius $R$, such that $z \in \overline{B(z)}$ and $z \in \overline{E(z)}$. The balls $B(z)$ and $E(z)$ are uniquely determined; their centers will be denoted by $b(z)$ and $e(z)$, respectively.

We will write $l(z)$ for the open line segment with end points $z$ and $b(z)$. Every point $y \in D$ such that $\delta_{D}(y)<R$ belongs to $l(z)$ for some $z \in \partial D$. Indeed, we may take $z \in \partial D$ with $|y-z|=\delta_{D}(y)$.

If $y \in l(z)$ for some $z \in \partial D$, then

$$
\delta_{D}(y)=\delta_{B(z)}(y)=\delta_{E(z)^{\mathrm{c}}}(y)=|y-z| .
$$


We fix $P=\left(p_{1}, \ldots, p_{d}\right) \in \partial D$ and put $p=\left(p_{1}, \ldots, p_{d-1}\right)$. To simplify the notation we assume as we may that there is a $C^{1,1}$ function $\varphi$ defined on $\left\{x \in \mathbb{R}^{d-1}:|p-x|<r_{0}\right\}$ such that $D \cap B\left(P, r_{0}\right)=\left\{\left(x_{1}, \ldots, x_{d-1}, x_{d}\right)\right.$ : $\left.x_{d}>\varphi\left(x_{1}, \ldots, x_{d-1}\right)\right\} \cap B\left(P, r_{0}\right)$ and, last but not least, $\nabla \varphi(p)=0$.

We take $Q \in \partial D \cap B\left(P, r_{0}\right)$. Again, we put $q=\left(q_{1}, \ldots, q_{d-1}\right)$, where $Q=\left(q_{1}, \ldots, q_{d-1}, q_{d}\right)$, so that $Q=(q, \varphi(q))$ and $P=(p, \varphi(p))$. Since $(-\nabla \varphi(q), 1)=\left(-\frac{\partial \varphi}{\partial x_{1}}(q), \ldots,-\frac{\partial \varphi}{\partial x_{d-1}}(q), 1\right)$ is a normal vector at $Q$,

$$
b(Q)=Q+R \frac{(-\nabla \varphi(q), 1)}{\sqrt{|\nabla \varphi(q)|^{2}+1}},
$$

which gives

$$
|b(Q)-b(P)| \leq(1+2 R \lambda)|Q-P|
$$

where $\lambda$ is the Lipschitz constant of $\nabla \varphi$. This shows that $\partial D \ni Q \mapsto b(Q) \in D$ is a Lipschitz function. The same argument works for the function $\partial D \ni$ $Q \mapsto e(Q) \in D^{\mathrm{c}}$.

We denote by $\langle\cdot, \cdot\rangle$ the usual inner product in $\mathbb{R}^{d}$ or $\mathbb{R}^{d-1}$. We have

$$
\begin{aligned}
|\langle b(Q)-Q, Q-P\rangle| & =\left|\left\langle R \frac{(-\nabla \varphi(q), 1)}{\sqrt{|\nabla \varphi(q)|^{2}+1}},(q-p, \varphi(q)-\varphi(p))\right\rangle\right| \\
& \leq \frac{R}{\sqrt{|\nabla \varphi(q)|^{2}+1}}(|\nabla \varphi(q)||q-p|+|\varphi(q)-\varphi(p)|) \\
& \leq \frac{R}{\sqrt{|\nabla \varphi(q)|^{2}+1}} 2 \lambda|q-p|^{2} \leq 2 R \lambda|Q-P|^{2},
\end{aligned}
$$

and so

$$
\begin{aligned}
|| b(Q)-\left.P\right|^{2}-R^{2} \mid & =|2\langle b(Q)-Q, Q-P\rangle+\langle Q-P, Q-P\rangle| \\
& \leq(4 R \lambda+1)|Q-P|^{2} .
\end{aligned}
$$

One of the main results of this paper is the following theorem.

Theorem 3.2. Let $x_{0} \in D$. For every $Q \in \partial D$ the limit

$$
g(Q)=\lim _{D \ni y \rightarrow Q} \frac{G_{D}\left(x_{0}, y\right)}{\delta_{D}(y)^{\alpha / 2}}
$$

exists and is a positive number. The function $g$ defined above is continuous on $\partial D$.

Proof. Fix $Q \in \partial D$. We first prove that

$$
g_{0}(Q)=\lim _{l(Q) \ni y \rightarrow Q} \frac{G_{D}\left(x_{0}, y\right)}{\delta_{D}(y)^{\alpha / 2}}
$$

exists and is a positive number. We consider $0<\eta \leq 1$ and let $x=x(Q)=$ 
$\eta b(Q)+(1-\eta) Q$. Let $l(Q) \ni y \rightarrow Q$. We have

$$
\begin{aligned}
\frac{G_{D}\left(x_{0}, y\right)}{\delta_{D}(y)^{\alpha / 2}} & =\frac{G_{D}\left(x_{0}, y\right)}{G_{D}(x, y)} \frac{G_{D}(x, y)}{\delta_{E(Q)^{\mathrm{c}}}(y)^{\alpha / 2}} \leq \frac{G_{D}\left(x_{0}, y\right)}{G_{D}(x, y)} \frac{G_{E(Q)^{\mathrm{c}}}(x, y)}{\delta_{E(Q)^{\mathrm{c}}}(y)^{\alpha / 2}} \\
& \leq \frac{G_{D}\left(x_{0}, y\right)}{G_{D}(x, y)} f_{R}(x-e(Q), y-e(Q)) \\
& \rightarrow M^{x_{0}}(x, Q)^{-1} f_{R}(x-e(Q), Q-e(Q))<\infty,
\end{aligned}
$$

and

$$
\begin{aligned}
\frac{G_{D}\left(x_{0}, y\right)}{\delta_{D}(y)^{\alpha / 2}} & \geq \frac{G_{D}\left(x_{0}, y\right)}{G_{D}(x, y)} \frac{f_{R}(x-b(Q), y-b(Q))}{\left(w\left(\frac{x-b(Q)}{R}, \frac{y-b(Q)}{R}\right)+1\right)^{d / 2}} \\
& \rightarrow M^{x_{0}}(x, Q)^{-1} f_{R}(x-b(Q), Q-b(Q))>0 .
\end{aligned}
$$

Therefore the limit (9), if exists, is positive and finite. Since

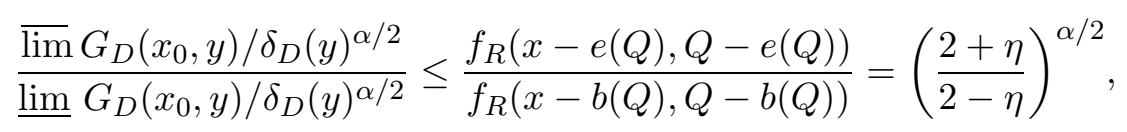

by letting $\eta \rightarrow 0$ we get the existence of the limit (9).

For $Q^{\prime} \in \partial D$ we have

$$
\left(\frac{2-\eta}{2+\eta}\right)^{\alpha / 2} \frac{M^{x_{0}}(x(Q), Q)}{M^{x_{0}}\left(x\left(Q^{\prime}\right), Q^{\prime}\right)} \leq \frac{g_{0}\left(Q^{\prime}\right)}{g_{0}(Q)} \leq\left(\frac{2+\eta}{2-\eta}\right)^{\alpha / 2} \frac{M^{x_{0}}(x(Q), Q)}{M^{x_{0}}\left(x\left(Q^{\prime}\right), Q^{\prime}\right)}
$$

and since the kernel $M^{x_{0}}(\cdot, \cdot)$ is continuous in $D \times \partial D$, and so is the function $\partial D \ni Q \mapsto x(Q) \in D$, the continuity of $g_{0}$ follows by letting $\eta \rightarrow 0$.

Now, let $y \in D$ be arbitrary and let $y \rightarrow Q$. Let $Q^{\prime} \in \partial D$ be such that $y \in l\left(Q^{\prime}\right)$. As in (10) we have

$$
\begin{aligned}
\frac{G_{D}\left(x_{0}, y\right)}{\delta_{D}(y)^{\alpha / 2}} & \leq \frac{G_{D}\left(x_{0}, y\right)}{G_{D}(x(Q), y)} \frac{G_{D}(x(Q), y)}{G_{D}\left(x\left(Q^{\prime}\right), y\right)} f_{R}\left(x\left(Q^{\prime}\right)-e\left(Q^{\prime}\right), y-e\left(Q^{\prime}\right)\right) \\
& \rightarrow M^{x_{0}}(x(Q), Q)^{-1} f_{R}(x(Q)-e(Q), Q-e(Q)) .
\end{aligned}
$$

The fact that $\lim _{y \rightarrow Q} G_{D}(x(Q), y) / G_{D}\left(x\left(Q^{\prime}\right), y\right)=1$, used above, follows from $\alpha$-harmonicity of $G_{D}(\cdot, y)$ in a ball centered at $x(Q)$ and (4), or from the gradient estimates of $[\mathrm{BKN}]$. We obtain similarly

$$
\varliminf_{y \rightarrow Q} \frac{G_{D}\left(x_{0}, y\right)}{\delta_{D}(y)^{\alpha / 2}} \geq M^{x_{0}}(x(Q), Q)^{-1} f_{R}(x(Q)-b(Q), Q-b(Q))
$$

and we proceed as before.

4. Convergence of $\alpha$-harmonic functions. We fix a point $x_{0} \in D$ and denote by $\sigma$ the $(d-1)$-dimensional Hausdorff measure on $\partial D$. We write $\delta_{x}$ for $\delta_{D}(x)$. 
Recall that the Lebesgue set of a function $f \in L^{1}(\partial D, \sigma)$ is the set of all $P \in \partial D$ satisfying

$$
\lim _{r \rightarrow 0^{+}} \frac{\int_{\partial D \cap B(P, r)}|f(Q)-f(P)| \sigma(d Q)}{\sigma(\partial D \cap B(P, r))}=0 .
$$

In particular

$$
\sup _{r>0} \frac{\int_{\partial D \cap B(P, r)}|f(Q)| \sigma(d Q)}{\sigma(\partial D \cap B(P, r))}<\infty
$$

for all points $P$ in the Lebesgue set of such an $f$. It is well known that the set of Lebesgue points is of full measure $\sigma$.

We introduce a suitable normalizing function:

$$
N(x)=\int_{\partial D} M^{x_{0}}(x, Q) \sigma(d Q) .
$$

By a remark after (6), $N$ is singular $\alpha$-harmonic on $D$, and this function will play a role of Doob's $h$ function as discussed in the introduction.

There are constants $c_{1}$ and $c_{2}$, depending only on $D, \alpha$ and $x_{0}$, such that

$$
0<c_{1} \leq \frac{N(x)}{\delta_{x}^{\alpha / 2-1}} \leq c_{2}<\infty
$$

for all $x \in D$. The proof of this fact is similar to the proof of Lemma 4.1 but simpler, and is left to the reader.

LEMmA 4.1. If $P \in \partial D$ is a Lebesgue point of $f \in L^{1}(\partial D, \sigma)$, then

$$
\lim _{D \ni x \rightarrow P} \int_{\partial D \cap\left\{Q:|Q-P| \geq|x-P|^{2 / 3}\right\}} M^{x_{0}}(x, Q) \delta_{x}^{1-\alpha / 2} f(Q) \sigma(d Q)=0 .
$$

Proof. We may assume $f \geq 0$. We fix $P \in \partial D$ from the Lebesgue set of $f$. Let $D \ni x \rightarrow P$. We put $B_{n}=B\left(P, 2^{n}|x-P|^{2 / 3}\right)$ and $A_{n}=B_{n} \backslash B_{n-1}$ for $n=1,2, \ldots$ Our assumptions on $D$ imply $\sigma\left(\partial D \cap B_{n}\right) \leq c\left(2^{n}|x-P|^{2 / 3}\right)^{d-1}$ for some constant $c$ depending only on $D$. By (5) we have

$\partial D \cap\left\{Q:|Q-P| \geq|x-P|^{2 / 3}\right\}$

$$
M^{x_{0}}(x, Q) \delta_{x}^{1-\alpha / 2} f(Q) \sigma(d Q)
$$

$$
\begin{aligned}
& \leq c^{\prime} \sum_{n=1}^{\infty} \int_{\partial D \cap A_{n}} \frac{\delta_{x}^{\alpha / 2}}{|x-Q|^{d}} \delta_{x}^{1-\alpha / 2} f(Q) \sigma(d Q) \\
& \leq c^{\prime} \delta_{x} \sum_{n=1}^{\infty}\left(\frac{1}{4} 2^{n}|x-P|^{2 / 3}\right)^{-d} \frac{\int_{\partial D \cap B_{n}} f(Q) \sigma(d Q)}{\sigma\left(\partial D \cap B_{n}\right)} c\left(2^{n}|x-P|^{2 / 3}\right)^{d-1} \\
& \leq c^{\prime \prime} \delta_{x}|x-P|^{-2 / 3} \leq c^{\prime \prime}|x-P|^{1 / 3} \rightarrow 0
\end{aligned}
$$

and the lemma follows. 
For $f \in L^{1}(\partial D, \sigma)$ we put

(cf. (6)).

$$
u(x)=\int_{\partial D} M^{x_{0}}(x, Q) f(Q) \sigma(d Q)
$$

TheOREM 4.2. Let $f \in L^{1}(\partial D, \sigma)$. If $f$ is continuous at $P \in \partial D$ then

$$
\lim _{x \rightarrow P} \frac{u(x)}{N(x)}=f(P)
$$

Generally, (12) holds at every Lebesgue point $P$ of $f$, but the limit should be taken nontangentially.

Proof. Let $P$ be a Lebesgue point of $f$. We have

$$
\begin{aligned}
\left|\frac{u(x)}{N(x)}-f(P)\right| & \\
\leq & \frac{\int_{\partial D \cap\left\{Q:|Q-P| \geq|x-P|^{2 / 3}\right\}} M^{x_{0}}(x, Q) \delta_{x}^{1-\alpha / 2}|f(Q)-f(P)| \sigma(d Q)}{\delta_{x}^{1-\alpha / 2} N(x)} \\
& +\frac{\int_{\partial D \cap\left\{Q:|Q-P|<|x-P|^{2 / 3}\right\}} M^{x_{0}}(x, Q)|f(Q)-f(P)| \sigma(d Q)}{N(x)} \\
= & I_{1}(x)+I_{2}(x) .
\end{aligned}
$$

From Lemma 4.1 with $|f(\cdot)-f(Q)|$ in place of $f$ and (11) it follows that $\lim _{x \rightarrow P} I_{1}(x)=0$.

Put $B_{n}=B\left(P, 2^{n}|x-P|\right)$ and $A_{n}=B_{n+1} \backslash B_{n}$ for $n \geq 1, A_{0}=B_{1}$. Denote by $n_{0}$ the smallest integer for which $B\left(P,|x-P|^{2 / 3}\right) \subset B_{n_{0}}$, so $-\frac{1}{3} \log _{2}|x-P| \leq n_{0}<-\frac{1}{3} \log _{2}|x-P|+1$. Note that $|x-Q| \geq \frac{1}{2} \delta_{x} 2^{n}$ for $Q \in A_{n}$. By (5) we have

$$
\begin{aligned}
N(x) I_{2}(x) & \leq c \delta_{x}^{\alpha / 2} \sum_{n=0}^{n_{0}} \int_{\partial D \cap A_{n}} \frac{|f(Q)-f(P)|}{|x-Q|^{d}} \sigma(d Q) \\
& \leq c^{\prime} \delta_{x}^{\alpha / 2} \sum_{n=0}^{n_{0}}\left(\delta_{x} 2^{n}\right)^{-d} \frac{\int_{\partial D \cap B_{n+1}}|f(Q)-f(P)| \sigma(d Q)}{\sigma\left(\partial D \cap B_{n+1}\right)}\left(2^{n}|x-P|\right)^{d-1} \\
& \leq c^{\prime} \delta_{x}^{\alpha / 2-d}|x-P|^{d-1} \sup _{0<r<2|x-P|^{2 / 3}} \frac{\int_{\partial D \cap B(P, r)}|f(Q)-f(P)| \sigma(d Q)}{\sigma(\partial D \cap B(P, r))} .
\end{aligned}
$$

From $(11), N(x) \geq c_{1} \delta_{x}^{\alpha / 2-1}$, so

$$
I_{2}(x) \leq c^{\prime \prime} \delta_{x}^{1-d}|x-P|^{d-1} \sup _{0<r<2|x-P|^{2 / 3}} \frac{\int_{\partial D \cap B(P, r)}|f(Q)-f(P)| \sigma(d Q)}{\sigma(\partial D \cap B(P, r))} .
$$

If $x \rightarrow P$ nontangentially then $\delta_{x} \geq \gamma|x-P|$ for some $\gamma>0$ and consequently $I_{2}(x) \rightarrow 0$. 
Moreover, if $f \in C(\partial D)$, then

$$
I_{2}(x) \leq \sup \left\{|f(Q)-f(P)|: Q \in \partial D,|Q-P|<|x-P|^{2 / 3}\right\} \rightarrow 0
$$

as $x \rightarrow P$. The proof is complete.

The exact asymptotics of $N(x)$ for $x$ near the boundary is described in the following

THEOREM 4.3. The limit

$$
\nu(P)=\lim _{D \ni x \rightarrow P} \frac{N(x)}{\delta_{x}^{\alpha / 2-1}}
$$

exists for all $P \in \partial D$. The function $\nu$ defined above is continuous on $\partial D$.

Proof. The proof is very similar to that of Theorem 3.2, however, it is more complicated. Fix $P \in \partial D$. We start by proving the existence of the limit

$$
\nu_{0}(P)=\lim _{l(P) \ni x \rightarrow P} N(x) \delta_{x}^{1-\alpha / 2}
$$

(see also (11)). Let $x \in l(P)$; then $\delta_{x}=|x-P|$.

Fix $0<\eta \leq 1$ and let $x_{1}=x_{1}(P)=\eta b(P)+(1-\eta) P$. We define $A(P, x)=\left\{Q \in \partial D:|Q-P|<|x-P|^{2 / 3}\right\}$. Taking $f \equiv 1$ in Lemma 4.1 we see that

$$
\lim _{l(P) \ni x \rightarrow P} N(x) \delta_{x}^{1-\alpha / 2}=\lim _{l(P) \ni x \rightarrow P} \int_{A(P, x)} M^{x_{0}}(x, Q) \delta_{x}^{1-\alpha / 2} \sigma(d Q)
$$

if either limit exists. From now on we assume $Q \in A(P, x)$. We have

$$
\begin{aligned}
|x-b(Q)|^{2}-R^{2}= & \langle x-P+P-b(Q), x-P+P-b(Q)\rangle-R^{2} \\
= & |x-P|^{2}+2\langle x-P, P-b(P)+b(P)-b(Q)\rangle \\
& +|P-b(Q)|^{2}-R^{2} \\
= & |x-P|^{2}-2 R|x-P|+2\langle x-P, b(P)-b(Q)\rangle \\
& +|P-b(Q)|^{2}-R^{2}
\end{aligned}
$$

and similarly

$$
\begin{aligned}
|x-e(Q)|^{2}-R^{2}= & |x-P|^{2}+2 R|x-P|+2\langle x-P, e(P)-e(Q)\rangle \\
& +|P-e(Q)|^{2}-R^{2} .
\end{aligned}
$$

From (7) and (8) we see that

$$
|\langle x-P, b(P)-b(Q)\rangle| \leq(1+2 R \lambda)|x-P|^{5 / 3}
$$

and

$$
|| P-\left.b(Q)\right|^{2}-R^{2}|\leq(4 R \lambda+1)| x-\left.P\right|^{4 / 3},
$$

hence for $x \in l(P)$ close enough to $P$ we have

$$
2 R(1-\eta)|x-P| \leq R^{2}-|x-b(Q)|^{2} \leq 2 R(1+\eta)|x-P|,
$$




$$
2 R(1-\eta)|x-P| \leq|x-e(Q)|^{2}-R^{2} \leq 2 R(1+\eta)|x-P| .
$$

In particular, $x, x_{1} \in B(Q)$.

Note that $M^{x_{0}}(x, Q)=M^{x_{0}}\left(x_{1}, Q\right) M^{x_{1}}(x, Q)$. By Lemma 3.1 and (15) we have

$$
\begin{aligned}
M^{x_{1}}(x, Q) & =\lim _{l(Q) \ni y \rightarrow Q} \frac{G_{D}(x, y)}{G_{D}\left(x_{1}, y\right)} \\
& \leq \varlimsup_{l(Q) \ni y \rightarrow Q} \frac{G_{E(Q)^{\mathrm{c}}}(x, y)}{\delta_{E(Q)^{\mathrm{c}}}(y)^{\alpha / 2}} \frac{\delta_{B(Q)}(y)^{\alpha / 2}}{G_{B(Q)}\left(x_{1}, y\right)} \\
& =\frac{|| x-\left.e(Q)\right|^{2}-\left.R^{2}\right|^{\alpha / 2}|x-Q|^{-d}}{|| x_{1}-\left.b(Q)\right|^{2}-\left.R^{2}\right|^{\alpha / 2}\left|x_{1}-Q\right|^{-d}} \\
& \leq \frac{(2 R(1+\eta)|x-P|)^{\alpha / 2}|x-Q|^{-d}}{|| x_{1}-\left.b(Q)\right|^{2}-\left.R^{2}\right|^{\alpha / 2}\left|x_{1}-Q\right|^{-d}}
\end{aligned}
$$

and similarly by Lemma 3.1 and (14),

$$
M^{x_{1}}(x, Q) \geq \frac{(2 R(1-\eta)|x-P|)^{\alpha / 2}|x-Q|^{-d}}{|| x_{1}-\left.e(Q)\right|^{2}-\left.R^{2}\right|^{\alpha / 2}\left|x_{1}-Q\right|^{-d}} .
$$

This gives

$$
\begin{aligned}
& \int_{A(P, x)} \frac{M^{x_{0}}(x, Q)}{\delta_{x}^{\alpha / 2-1}} \sigma(d Q) \\
& \leq \delta_{x} \int_{A(P, x)} \frac{(2 R(1+\eta))^{\alpha / 2}|x-Q|^{-d} M^{x_{0}}\left(x_{1}, Q\right)}{|| x_{1}-\left.b(Q)\right|^{2}-\left.R^{2}\right|^{\alpha / 2}\left|x_{1}-Q\right|^{-d}} \sigma(d Q) \\
& \leq \sup _{Q \in A(P, x)} \frac{(2 R(1+\eta))^{\alpha / 2}\left|x_{1}-Q\right|^{d}}{|| x_{1}-\left.b(Q)\right|^{2}-\left.R^{2}\right|^{\alpha / 2}} \\
& \quad \times \sup _{Q \in A(P, x)} M^{x_{0}}\left(x_{1}, Q\right) \cdot \delta_{x} \int_{A(P, x)}|x-Q|^{-d} \sigma(d Q) \\
& \rightarrow\left(\frac{2(1+\eta)}{\eta(2-\eta)}\right)^{\alpha / 2}(\eta R)^{d} \cdot M^{x_{0}}\left(x_{1}, P\right) \cdot \int_{\mathbb{R}^{d-1}}\left(|q|^{2}+1\right)^{-d} d q
\end{aligned}
$$

as $x \rightarrow P$. Similarly we obtain

$$
\begin{aligned}
\varliminf_{l(P) \ni x \rightarrow P} \int_{A(P, x)} \frac{M^{x_{0}}(x, Q)}{\delta_{x}^{\alpha / 2-1}} \sigma(d Q) \geq & \left(\frac{2(1-\eta)}{\eta(2+\eta)}\right)^{\alpha / 2}(\eta R)^{d} \cdot M^{x_{0}}\left(x_{1}, P\right) \\
& \times \int_{\mathbb{R}^{d-1}}\left(|q|^{2}+1\right)^{-d} d q .
\end{aligned}
$$


Thus

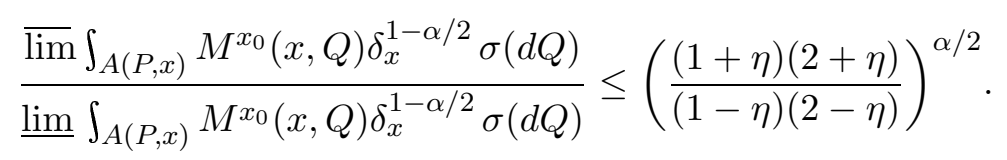

Since $\eta>0$ can be arbitrarily small, the existence of the limit (13) follows.

The rest of the proof is very similar to the corresponding part of the proof of Theorem 3.2 and will be omitted.

Corollary 4.4. Let $f \in L^{1}(\partial D, \sigma)$. If $f$ is continuous at $P \in \partial D$ then

$$
\lim _{x \rightarrow P} \frac{u(x)}{\delta_{x}^{\alpha / 2-1}}=\nu(P) f(P)
$$

Generally, (16) holds at every Lebesgue point $P$ of $f$, but the limit should be taken nontangentially.

EXAMPLE 4.5. Let $D$ be the ball $B(0, r) \subset \mathbb{R}^{d}, r>0$, and $x_{0}=0$. The corresponding Martin kernel $M$ is

$$
M(x, Q)=r^{d-\alpha} \frac{\left(r^{2}-|x|^{2}\right)^{\alpha / 2}}{|x-Q|^{d}}, \quad|x|<r,
$$

for all $Q \in \partial B(0, r)$. Formula (17) is an exact analogue of the classical Poisson kernel for the ball. It follows easily that multiplication by $r^{2-\alpha}\left(r^{2}-|x|^{2}\right)^{\alpha / 2-1}$ is an isomorphism from the nonnegative functions harmonic in $B(0, r)$ onto the nonnegative singular $\alpha$-harmonic functions in $B(0, r)$. Clearly, there is no such isomorphism for general $C^{1,1}$ domains, as can be seen by considering a disconnected domain $D$.

We note that Theorem 4.2 readily generalizes to other normalizing functions $\widetilde{N}(x)=\int_{\partial D} M^{x_{0}}(x, Q) r(Q) \sigma(d Q)$ for nonnegative $r \in L^{1}(\partial D, \sigma)$ by using (12) twice. Further generalization in the spirit of [Wu1] requires a separate argument and should perhaps be considered in the more general context of Lipschitz domains.

\section{References}

[A] A. Ancona, Principe de Harnack à la frontière et théorème de Fatou pour un opérateur elliptique dans un domaine lipschitzien, Ann. Inst. Fourier (Grenoble) 28 (1978), no. 4, 169-213.

[BM] R. Bañuelos and C. N. Moore, Probabilistic Behavior of Harmonic Functions, Birkhäuser, Basel, 1999.

[Ba] R. F. Bass, Probabilistic Techniques in Analysis, Springer, New York, 1995.

[BaB] R. F. Bass and K. Burdzy, A probabilistic proof of the boundary Harnack principle in: Seminar on Stochastic Processes, 1989 (San Diego, CA, 1989), Birkhäuser Boston, Boston, MA, 1990, 1-16.

$[\mathrm{BaC}] \quad$ R. F. Bass and M. Cranston, Exit times for symmetric stable processes in $\mathbb{R}^{n}$, Ann. Probab. 11 (1983), 578-588. 
[BG] R. M. Blumenthal and R. K. Getoor, Markov Processes and Potential Theory, Springer, New York, 1968.

[BGR] R. M. Blumenthal, R. K. Getoor and D. B. Ray, On the distribution of first hits for the symmetric stable processes, Trans. Amer. Math. Soc. 99 (1961), 540-554.

[B1] K. Bogdan, The boundary Harnack principle for the fractional Laplacian, Studia Math. 123 (1997), 43-80.

[B2] -, Representation of $\alpha$-harmonic functions in Lipschitz domains, Hiroshima Math. J. 29 (1999), 227-243.

[B3] -, Sharp estimates for the Green function in Lipschitz domains, J. Math. Anal. Appl. 243 (2000), 326-337.

[BBC] K. Bogdan, K. Burdzy and Z. Q. Chen, Censored stable processes, preprint, 2001.

[BB1] K. Bogdan and T. Byczkowski, Probabilistic proof of boundary Harnack principle for $\alpha$-harmonic functions, Potential Anal. 11 (1999), 135-156.

[BB2] - - - Potential theory for the $\alpha$-stable Schrödinger operator on bounded Lipschitz domains, Studia Math. 133 (1999), 53-92.

[BB3] - - - On the Schrödinger operator based on the fractional Laplacian, Bull. Polish Acad. Sci. Math. 49 (2001), 291-301.

[BKN] K. Bogdan, T. Kulczycki and A. Nowak, Gradient estimates for harmonic and q-harmonic functions of symmetric stable processes, Illinois J. Math. 46 (2002), 541-556.

[Ca] L. Carleson, Selected Problems on Exceptional Sets, Van Nostrand, London, 1967.

[CS1] Z. Q. Chen and R. Song, Estimates on Green function and Poisson kernels for symmetric stable process, Math. Ann. 312 (1998), 465-501.

[CS2] -, - Correction: Estimates on Green function and Poisson kernels for symmetric stable process, 1999.

[CS3] - - - Martin boundary and integral representation for harmonic functions of symmetric stable processes, J. Funct. Anal. 159 (1998), 267-294.

[CZ] K. L. Chung and Z. X. Zhao, From Brownian Motion to Schrödinger's Equation, Springer, Berlin, 1995.

[Da] B. Dahlberg, Estimates of harmonic measure, Arch. Rational Mech. Anal. 65 (1977), 275-288.

[D1] J. L. Doob, A relativized Fatou theorem, Proc. Nat. Acad. Sci. U.S.A. 45 (1959), 215-222.

[D2] - Classical Potential Theory and its Probabilistic Counterpart, Springer, New York, 1984.

[F] P. Fatou, Séries trigonométriques et séries de Taylor, Acta Math. 30 (1906), 335-400.

$[\mathrm{J}] \quad$ T. Jakubowski, The estimates for the Green function in Lipschitz domains for the symmetric stable processes, preprint, 2002.

[JK] D. S. Jerison and C. E. Kenig, Boundary value problems on Lipschitz domains, in: Studies in Partial Differential Equations, W. Littman (ed.), MAA Stud. Math. 23, Math. Assoc. Amer., 1982, 1-68.

[K] T. Kulczycki, Properties of Green function of symmetric stable processes, Probab. Math. Statist. 17 (1997), 381-406.

[L] N. S. Landkof, Foundations of Modern Potential Theory, Springer, New York, 1972.

[M] P. J. Méndez-Hernández, Exit times from cones in $\mathbb{R}^{n}$ of symmetric stable processes, Illinois J. Math. 46 (2000), 155-163.

[MS] K. Michalik and K. Samotij, Martin representation for $\alpha$-harmonic functions, Probab. Math. Statist. 20 (2000), 75-91. 
[Mi] P. W. Millar, First passage distributions of processes with independent increments, Ann. Probab. 3 (1975), 215-233.

[R1] M. Riesz, Intégrales de Riemann-Liouville et potentiels, Acta Sci. Math. (Szeged) 9 (1938), 1-42.

[R2] -, Rectification au travail "Intégrales de Riemann-Liouville et potentiels", ibid., 116-118.

[S] E. M. Stein, Singular Integrals and Differentiability Properties of Functions, Princeton Univ. Press, Princeton, NJ, 1970.

[Sz] P. Sztonyk, On harmonic measure for Lévy processes, Probab. Math. Statist. 20 (2000), 383-390.

[Wu1] J.-M. Wu, Comparisons of kernel functions, boundary Harnack principle and relative Fatou theorem on Lipschitz domains, Ann. Inst. Fourier (Grenoble) 28 (1978), no. 4, 147-167.

[Wu2] -, Harmonic measures for symmetric stable processes, Studia Math. 149 (2002), 281-293.

Institute of Mathematics

Wrocław University of Technology

50-370 Wrocław, Poland

E-mail: bogdan@im.pwr.wroc.pl

bdyda@im.pwr.wroc.pl 